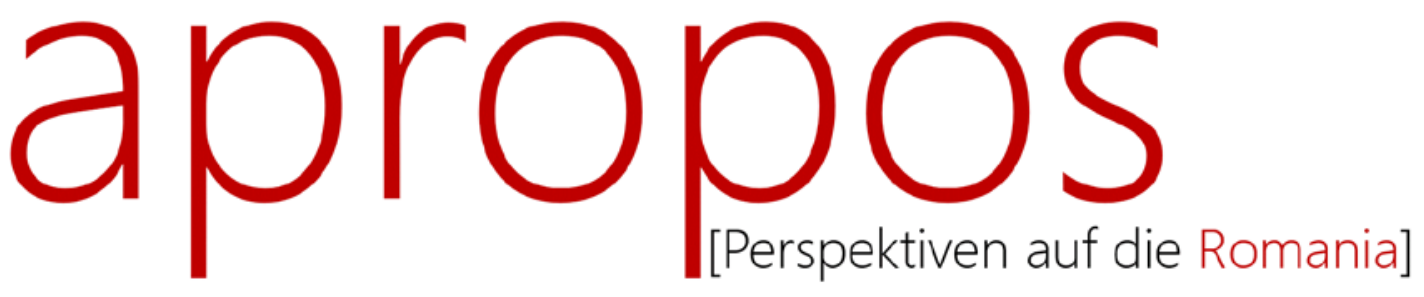

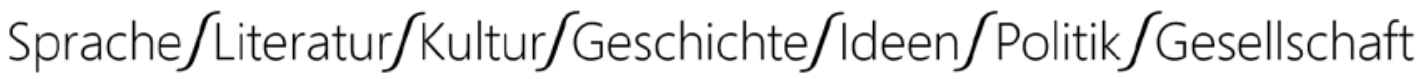

2 | 2019

Rugbykultur (in) der Romania

Rezension von Mauro, Max. 2016. The Balotelli

Generation. Issues of Inclusion and Belonging in Italian

Football and Society. Bern: Peter Lang.

Markus Grimm

apropos [Perspektiven auf die Romania]

hosted by Hamburg University Press

STAATS- UND UNIVERSITÄTS-

BIBLIOTHEK

H A M B UR G

2019, 2

CARL VON OSSIETZKY

pp. $153-155$

ISSN: 2627-3446

Online

https://journals.sub.uni-hamburg.de/apropos/article/view/1381

Zitierweise

Grimm, Markus. 2019. „Mauro, Max. 2016. The Balotelli Generation. Issues of Inclusion and Belonging in Italian Football and Society. Bern: Peter Lang. ", apropos [Perspektiven auf die Romania] 2, 153-155. doi: 10.15460/apropos.0.1381

Except where otherwise noted, this article is licensed under a Creative Commons Attribution 4.0 International license (CC BY 4.0)

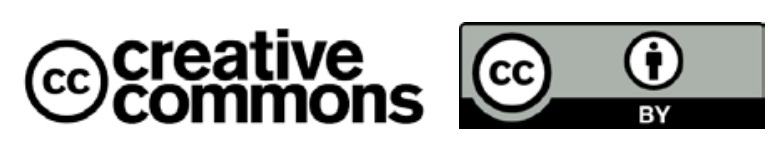


Markus Grimm

Rezension

\title{
MAURO, Max. 2016. The Balotelli Generation. Issues of Inclusion and Belonging in Italian Football and Society. Bern: Peter Lang.
}

Fußball als Spiegel oder Zerrbild der Gesellschaft? Die Rolle junger Immigranten im italienischen Fußball und die Potentiale des Sports als Mittel der Integration und Inklusion

\author{
Markus Grimm \\ ist Referent für Internationale \\ Aufgaben beim Zentralkomitee der \\ deutschen Katholiken und \\ langjähriges Mitglied von PIFO - \\ Politische Italienforschung an der \\ Justus-Liebig-Universität Gießen. \\ markus.grimm@zdk.de
}

Keywords

Integration - Fußball - Sport - Rassismus - Italien

Die Probleme eines Landes exemplarisch an einer Biografie zu analysieren ist ein gewagtes Unterfangen, das in diesem Fall allerdings hervorragend gelungen ist. Max Mauro nimmt die sportliche Karriere - mit allen ihren Höhen und Tiefen - des italienischen Fußball-Nationalspielers Mario Balotelli als roten Faden für die Suche nach der integrierenden Kraft des Sports und zugleich seinen hemmenden Faktoren. Dies gelingt, weil Mauro sich nicht an diesem einen Ausnahmefall festhält, sondern weil zahlreiche Interviews mit jungen Spielern, Trainern, Funktionären und anderen sportpolitischen Entscheidungs-trägern die empirische Grundlage bilden. Der „Fall Balotelli“ dient hier eher als Illustration mit anschaulichen Beispielen und dem Abgleich zwischen den Vorkommnissen in unteren Ligen und an der Spitze des italienischen Fußballs. Balotelli ist in Palermo als Sohn ghanaischer Eltern geboren, konnte aber erst mit seiner Volljährigkeit nach italienischem Recht einen italienischen Pass beantragen. Dadurch spielte er für keine nationale Jugendauswahl (die Angebote, für Ghana anzutreten, lehnte er ab) und wurde frisch eingebürgert sogleich in die U21-Nationalmannschaft berufen. Mit einer längeren Unterbrechung spielt er seit 2010 für die italienische Nationalmannschaft und seitdem, abgesehen von zwei kürzeren Engagements beim AC Milan, für englische und französische Vereine auf höchstem Niveau. 
Während in Deutschland bereits einige wissenschaftliche Beiträge zur (potentiellen) Integrationskraft des Sports erschienen sind (so z. B. schon 2011 von Sebastian Braun und Tina Nobis), ist dieses Thema in Italien weder wissenschaftlich noch in der Gesellschaft und besonders im Profisport nicht weit verbreitet. Max Mauro nähert sich diesem Thema mit dem Blick "von außen" und mit großer Leidenschaft für das Thema. Gleich zu Beginn macht er dieses mehr als wissenschaftliche Interesse transparent und beschreibt seine Motivation, mehr verstehen zu wollen über die Bedeutung, die Fußball (als die mit Abstand populärste Sportart in Italien und für viele Migranten) für junge Immigranten und ihr Zugehörigkeitsgefühl für die Gesellschaft hat. Ausdrücklich versteht es Mauro auch als seine Aufgabe, nicht nur über junge Migranten zu forschen, sondern diesen eine Stimme zu geben und die Situation und Verortung der Migranten in Italien aus einer geradezu ethnographischen Innensicht zu berichten.

Während in der Serie A, der ersten italienischen Fußballliga der Männer, neben den ausländischen Stars auch zahlreiche in Italien aufgewachsene Migranten spielen, ist deren Präsenz in der italienischen Nationalmannschaft noch gering. Es gibt daher weniger Vorbilder (wie Balotelli) für den Weg an Spitze als etwa in Deutschland, das hier etwa eine Generation voraus ist und bei Mauro als Vorbild Erwähnung findet.

Die Arbeit beruht auf einer ausgewogenen Mischung aus literaturtheoretischen Grundlagen, der Analyse offizieller Dokumente und besonders einer qualitativen Studie aus über 70 Tiefeninterviews. In den theoretischen Grundlagen beschäftigt sich Max Mauro mit der Klärung der zugrunde liegenden Terminologien rund um Inklusion und Zugehörigkeit und den korrespondierenden Konzepten (Kapitel 1) sowie den historischen Grundlagen der Arbeit (Kapitel 2). Hier beschreibt Mauro die Geschichte des italienischen Fußballs mit Blick auf dessen nationales und nationalistisches Potential und belegt mit wenigen klaren Zahlen die Schwierigkeiten, die eine stärkere Beteiligung von Migranten und der ersten Generation im Land geborener Kinder von Migranten in Italien verhindern.

Im empirischen Teil der Arbeit beschreibt und analysiert Mauro, wie stark Diskriminierung den Aufstieg von Immigranten im Fußball (und der Gesellschaft) prägt und Integration behindert. Die Ursache sieht der Autor in einem Zusammenspiel aus tradierten rassistischen Einstellungen, überbordender Regulierung von Seiten des nationalen Fußballverbands FIGC und der Dachverbände UEFA und FIFA. Gerade für die diskriminierende Haltung vieler Spieler und Funktionäre finden sich zahllose Beispiele, die von der Serie A bis in die untersten Freizeitligen zu finden sind und für die sich auch gute Beispiele in der Karriere des Vorbilds Balotelli finden. Die Monographie schließt aber mit einem optimistischen stimmenden Ausblick auf zwei jüngere Entwicklungen des italienischen Fußballs: zum einen die Einführung eines Gesetzes zu einer Sport-Staatsbürgerschaft, welches die Einbürgerung von in Italien lebenden Sportlern ermöglicht bzw. die Möglichkeit schafft, dass Jugendliche ohne italienischen Pass, aber mit dauerhaftem Wohnsitz in Italien seit dem 10. Lebensjahr, wie EU-Bürger im Sport behandelt werden können; zum anderen die Herausbildung einer parallelen Amateursportbewegung, die unter weniger strikter Regulierung sportliche Wettkämpfe ermöglicht und damit für Migranten leichter zugänglich ist und diesen 
somit auch eine Gelegenheit bietet, sich jenseits der etablierten Strukturen für den Profisport zu präsentieren. Mauro kritisiert an der extrem streng ausgelegten und angewendeten Regelsetzung, welche gerade im Jugendbereich in der Theorie Menschenhandel und Ausbeutung verhindern soll, dass diese in der Praxis zu Exklusion führt, weil die Integration von Nicht-EU-Bürgern in der professionellen Ausbildung so verhindert wird. Wenn es nicht möglich ist, Jugendlichen ohne italienischen (oder europäischen) Pass eine Ausbildung in einem Fußballinternat zu ermöglichen, während ein Stipendium für ein normales Internat durchaus möglich wäre, sieht Mauro hier eine Ungleichbehandlung der "Fußball-Industrie" gegenüber anderen ökonomischen Bereichen.

Im Untertitel klingt bereits an, dass Mauro den Fußball als Spiegel der Gesellschaft betrachtet und die italienische Gesellschaft über ihre Leidenschaft für den calcio zu verstehen versucht. Man sollte hierbei jedoch auch nicht vergessen, dass weder der Profifußball noch die korrespondierende Fankultur zwingend ein repräsentativer Querschnitt der Bevölkerung sind. Fehlendende Wechselwirkungen und Reaktionen der Öffentlichkeit auf Ereignisse im Fußball (wie rassistische Beleidigungen) lassen auch die Vermutung zu, dass die Kultur des calcio auch ein verzerrtes Abbild sein könnte. Gerade in den letzten Jahren hat zum Beispiel der Anteil rechtsextremer Fangruppen an Dominanz gegenüber den linken und linksextremen Gruppierungen gewonnen. Auch wenn dies vordergründig mit der Schwäche der politischen Linken und dem Auftrieb für die Lega einhergeht, sollte man doch mit Parallelen vorsichtig sein. Auch das Gewaltpotential und die geringe Sicherheit in vielen Stadien trägt dazu bei, dass weite Teile der Bevölkerung nicht unter den klassischen Anhängern vertreten sind. Ein zweiter kleiner Kritikpunkt aus Sicht des Rezensenten ist die weitgehende Gleichsetzung von Fußball mit Leistungssport. Auch unter den Migranten gibt es viele, die Sport nicht als Mittel zu Aufstieg, Erfolg und Anerkennung verstehen, sondern als Breitensport ohne höhere Ambitionen betreiben. Jenseits dieser Anmerkungen ist das Buch, auch dank schöner, breit gestreuter und manchmal augenzwinkernder kulturhistorischer Belege, ein lesenswertes Werk zu Kraft und Grenzen der Integration durch Sport.

Der Autor der Monographie ist Mitarbeiter für Sport Studies an der Southampton Solent University in Großbritannien, die einen ausgewiesenen Schwerpunkt in den Sportwissenschaften besitzt. Max Mauro ist zudem gebürtiger Italiener mit einem Abschluss in Padua und einer Promotion in Irland. Die „Balotelli Generation“ wurde durch ein Havelange-Forschungsstipendium der FIFA gefördert, was erfreulicherweise nicht die kritische Haltung Max Mauros gegenüber den sportpolitischen Akteuren beeinflusst hat. Die profunde Kenntnis der Materie zeigt sich auch in der ausführlichen und gut zusammengestellten Bibliographie, die auch als Basis der Recherche für weitere Arbeiten zum Thema (auch über Italien hinaus) empfehlenswert ist. 